\title{
Antitumor Activity of $\alpha$-Linolenic Acid-Paclitaxel Conjugate Nanoparticles: In vitro and in vivo
}

\author{
Mei-Qi Xu ${ }^{1,2}$ \\ Yan-Li Hao ${ }^{1,2}$ \\ Jing-Ru Wang ${ }^{1,2}$ \\ Zhuo-Yue $\mathrm{Li}^{1,2}$ \\ Hui Li ${ }^{l}$ \\ Zhen-Han Feng ${ }^{1,2}$ \\ Hui Wang ${ }^{l, 2}$ \\ Jing-Wen Wang ${ }^{1,2}$ \\ Xuan Zhang iD ${ }^{1,2}$ \\ 'Beijing Key Laboratory of Molecular \\ Pharmaceutics and New Drug Delivery \\ Systems, School of Pharmaceutical \\ Sciences, Peking University, Beijing, \\ 100191, People's Republic of China; \\ ${ }^{2}$ Department of Pharmaceutics, School of \\ Pharmaceutical Sciences, Peking \\ University, Beijing, 10019I, People's \\ Republic of China
}

Purpose: Small molecule modified antitumor drug conjugate nanoparticles have the advantages of high drug loading, simple synthesis and preparation, and better biocompatibility. Due to the large demand for exogenous $\alpha$-linolenic acid (ALA) by tumor cells, we synthesized $\alpha$-linolenic acid-paclitaxel conjugate (ALA-PTX) and prepared $\alpha$-linolenic acidpaclitaxel conjugate nanoparticles (ALA-PTX NPs), in order to obtain better tumor cellular uptake and antitumor activity in vitro and in vivo.

Methods: We synthesized and characterized ALA-PTX, and then prepared and characterized ALA-PTX NPs. The cellular uptake, uptake pathways, intracellular behavior, in vitro and in vivo antitumor activity of ALA-PTX NPs were evaluated.

Results: The size of ALA-PTX NPs was approximately $110.7 \pm 1.7 \mathrm{~nm}$. The drug loading was approximately $90 \%(\mathrm{w} / \mathrm{w})$ with CrEL-free and organic solvent-free characteristics. The cellular uptake of ALA-PTX NPs was significantly higher than that of PTX injection by MCF-7, MCF-7/ADR and HepG2 cells. In these three cell lines, the cellular uptake of ALAPTX NPs at $6 \mathrm{~h}$ was approximately 1.5-2.6 times higher than that of PTX injection. ALAPTX NPs were ingested through clathrin-mediated endocytosis, then transferred to lysosomes, and could dissolve in cells to play an antitumor activity. The in vitro and in vivo antitumor activity of ALA-PTX NPs was confirmed in MCF-7/ADR and HepG2 cell models and tumor-bearing nude mouse models.

Conclusion: ALA-PTX NPs developed in our study could provide a new method for the preparation of nano-delivery systems suitable for antitumor therapy that could increase tumor cellular uptake and enhance antitumor activity.

Keywords: $\alpha$-linolenic acid, $\alpha$-linolenic acid-paclitaxel conjugate, $\alpha$-linolenic acid-paclitaxel conjugate nanoparticles, cellular uptake, antitumor activity

\section{Introduction}

The application of nanomedicine in cancer treatment has attracted considerable attention as a way to enhance tumor targets and reduce adverse effects. ${ }^{1,2}$ Many nanomaterials, such as organic, inorganic, lipid, protein or polymer, are used as carrier materials for constructing antitumor nanomedicine. ${ }^{3,4}$ However, in the process of research and application, some problems have also been found to limit its clinical conversion: burst release, low drug loading (usually less than 10\%), and systemic toxicity caused by a large number of carrier materials. ${ }^{5}$

The carrier-free NPs has opened up a new way for the construction of stable NPs and the development of antitumor drugs platforms. ${ }^{6-8}$ The carrier-free NPs have the higher delivery efficiency and the better safety. ${ }^{9-12}$ Many studies have constructed macromolecule-drug conjugate NPs, but there are still some problems
Correspondence: Xuan Zhang

Department of Pharmaceutics, School of

Pharmaceutical Sciences, Peking

University, Xueyuan Road 38, Beijing,

I00191, People's Republic of China

Tel +86-10-82805765

Fax +86-10-8280576

Email xuanzhang@bjmu.edu.cn 
including uneven drug loading and low occupancy of antitumor drugs. In order to overcome these shortcomings, small molecule modified antitumor drug conjugates with higher drug occupancy rates have been synthesized and these conjugates can form NPs in water. These NPs have a significantly high drug loading, simple synthesis and preparation, and better biocompatibility.

Polyunsaturated fatty acids (PUFAs) are essential components of cell membranes. In addition to its important physiological functions such as anti-inflammatory, maintaining cardiovascular health, and promoting brain development, it has also been found to have significant antitumor activity, which can increase the sensitivity of chemotherapeutic drugs, and shows great tumor treatment potential. $^{13,14}$ Therefore, these PUFAs can be considered ideal functional materials for modifying chemotherapy drugs. $^{9-11}$ In our previous research, we used conjugated linoleic acid (CLA) as a functional material to modify the chemotherapy drug paclitaxel (PTX) and synthesized conjugated linoleic acid-paclitaxel conjugate (CLA-PTX) which could cross the blood-brain barrier and target brain tumor. ${ }^{15}$ CLA-PTX can form stable nanoparticles in water. The cellular uptake of CLA-PTX NPs by tumor cells was significantly enhanced. ${ }^{16}$ CLA-PTX NPs have good stability, long-term circulation, and good antitumor activity. $^{16}$

Omega-3 PUFA have been shown to regulate cell growth and differentiation, anti-inflammatory, regulate immunity, and inhibit the growth and proliferation of cancer cells, invasion and angiogenesis. ${ }^{17-19}$ The latest research claimed that omega-3 PUFA may be the key to opening the blood-brain barrier. ${ }^{20}$ Omega-3 PUFA could be recognized by specialized transporters MFSD2A and promoted their crossing the blood-brain barrier. ${ }^{20}$ This ability to easily transmembrane may enable omega-3 PUFA modified drugs have higher uptake, which is helpful for the design of new drugs.

$\alpha$-Linolenic acid (ALA) is a kind of omega-3 PUFAs, that cannot be synthesized endogenously. Due to increased proliferation and metabolism, tumor cells are highly dependent on the uptake of exogenous ALA. ${ }^{21}$ Studies have shown that ALA can induce breast cancer cell apoptosis by inhibiting the HER2 signal transduction pathway. $^{22-24}$ Wang et al modified the 10-hydroxyl group of camptothecin derivatives (SN38) with ALA to obtain ALA-SN38, which can form stable NPs in water with good antitumor activity. ${ }^{9}$ Therefore, ALA is an ideal small molecule functional material for constructing small molecule modified antitumor drug conjugate NPs.

In the present research, we synthesized $\alpha$-linolenic acid-paclitaxel conjugate (ALA-PTX). ALA-PTX was characterized by NMR and mass spectrometry analysis. $\alpha$ Linolenic acid-paclitaxel conjugate nanoparticles (ALAPTX NPs) were then prepared and characterized. We investigated the cellular uptake and uptake pathways in MCF-7, MCF-7/ADR and HepG2 cell lines. The intracellular behavior of ALA-PTX NPs in the aspects of intracellular dissolution, co-localization with endocytic proteins, intracellular transport and efflux pathways were also investigated. The in vitro and in vivo antitumor activity of ALA-PTX NPs were evaluated.

\section{Materials and Methods Materials}

Paclitaxel (PTX) was obtained from Ouhe Co., Ltd. (Beijing, China). $\alpha$-linolenic acid (ALA) was supplied from SigmaAldrich (St. Louis, MO, USA). 1,2-distearoyl-sn-glycero -3-phosphoethanolamine [methoxy(polyethylene glycol) 2000] (DSPE-PEG) was purchased from NOF Co. (Tokyo, Japan). Paclitaxel injection was commercially available from local hospital of Beijing (Bristol Myers Squibb Co., Princeton, NJ, U.S.A.). Hypertonic sucrose, filipin, ethylisopropylamiloride (EIPA) and chlorpromazine (CPZ) were supplied from Sigma-Aldrich (St. Louis, MO, USA). AntiClathrin heavy chain antibody, anti-caveolin-1-N-terminal (Alexa Fluor 488) antibody and goat anti-Rabbit IgG H\&L (Alexa Fluor 647) were obtained from Abcam (Cambridge, MA, USA). Anti-Rab3A, anti-Rab8A, the dye DiI, DiO and Hoechst 33342 were purchased from Beyotime Biotechnology Co., Ltd. (Shanghai, China). LysoBrite ${ }^{\mathrm{TM}}$ NIR were obtained from AAT Bioquest (Sunnyvale, CA). All other chemicals were of analytical or HPLC grade.

Cell culture medium, RPMI 1640 and DMEM medium were obtained from Macgene Biotech Co. Ltd. (Beijing China). Fetal bovine serum (FBS) was supplied from GIBCO (Invitrogen Co. (Carlsbad, USA)).

\section{Cell Lines}

Human breast carcinoma MCF-7 cells were obtained from the Cell Resource Center, Peking Union Medical College (Beijing, China). Drug-resistant human breast carcinoma MCF-7/ADR cells and human hepatocellular carcinoma HepG2 were supplied from the Chinese Academy of Sciences Cell Bank (Shanghai, People's Republic of 
China). Cells were cultured according to the recommended conditions in ATCC.

\section{Animals}

Female BALB/C nude mice (4-6 weeks) were obtained from the Experimental Animal Center of Peking University Health Science Center. All care and handling of the animals were performed with the approval of the Experimental Animal Center of Peking University Health Science Center. Additionally, this study was performed following the National Institutes of Health guidelines for the use of experimental animals.

\section{Synthesis of ALA-PTX}

ALA-PTX was synthesized according to our previous PTX conjugate method. ${ }^{16}$ Briefly, ALA-PTX was synthesized through a direct esterification reaction on the $\mathrm{C} 2$ '-hydroxyl of PTX catalyzed by DCC/DMAP (Sigma-Aldrich, St. Louis, MO, USA). Then, the reaction mixture was filtered to remove N,N'-dicyclohexylurea (DCU), and the filtrate was dried by rotary evaporation. The crude product was purified by silica-gel column chromatography and eluted with petroleum ether/acetone (gradually decreasing from 20:1 to $3: 1, \mathrm{v} / \mathrm{v}$ ). The resulting eluent was dried by rotary evaporation to obtain ALA-PTX.

\section{Characterization of ALA-PTX 'H NMR Spectra}

ALA-PTX was dissolved in deuterated chloroform and determined by nuclear magnetic resonance spectroscopy (400 MHz ${ }^{1} \mathrm{H}$ NMR, Bruker AVANCEZ 400).

\section{Mass Spectrometry}

ALA-PTX was characterized by mass spectrometry (MALDI-TOF-MS, Waters).

\section{HPLC Analysis}

The purity of ALA-PTX was determined by highperformance liquid chromatography (HPLC) according to our previous HPLC method using an LC-20AT liquid chromatograph (SHIMADZU, Japan) and SPD-M20A diode array detector (SHIMADZU, Japan). The details of the HPLC method are shown in our previous report, ${ }^{16,25}$ as shown in Table S1.

\section{Preparation of ALA-PTX NPs}

ALA-PTX NPs were prepared using a simple precipitation method. ${ }^{16,25,26}$ In brief, ALA-PTX and DSPE-PEG (ALA-
PTX: DSPE-PEG = 1: 0.1, w/w) were dissolved in DMSO. A volume of $1 \mathrm{~mL}$ of the above solution was added dropwise into distilled water $(3 \mathrm{~mL})$ with stirring $(300$ $500 \mathrm{rpm})$ at room temperature. Then, ALA-PTX NPs formed spontaneously. The resulting NP dispersion was dialyzed against distilled water for $24 \mathrm{~h}$ (MWCO $=3500$ Da), and the obtained NP dispersion was subjected to ultrafiltration at $4000 \mathrm{rpm}$ for $5 \mathrm{~min}$.

For preparation of DiI-loaded ALA-PTX NPs, ALAPTX, DSPE-PEG and the fluorescent dye DiI (100:10:5, $\mathrm{w} / \mathrm{w})$ were dissolved in DMSO. Then, a procedure similar to that used for the preparation of ALA-PTX NPs was performed.

DiO\&DiI- loaded ALA-PTX NPs were prepared by slowly adding the stock solution with the drug mass ratio: ALA-PTX:DSPE-PEG:DiO:DiI $=100: 10: 2.5: 2.5$ to 10 times the volume of distilled water under constant stirring under constant stirring.

\section{Characterization of ALA-PTX NPs}

\section{Particle Size and Zeta Potential}

The particle size and zeta potential of ALA-PTX NPs were measured by a dynamic light scattering detector (Malvern, Worcestershire, UK).

\section{Transmission Electron Microscopy (TEM)}

The morphology of ALA-PTX NPs was characterized by TEM (JEM-1400Plus, JEOL, Japan).

\section{Fourier Transform Infrared Spectroscopy (FTIR)}

The lyophilized powder of ALA-PTX NPs was characterized by FT-IR measurements recorded using a Fourier transform infrared spectrometer (Nicolet-6700, Thermo Fisher, USA).

\section{In vitro Cellular Uptake}

MCF-7, MCF-7/ADR or HepG2 cells were seeded in a 12 -well plate at a density of $2 \times 10^{5}$ cells/well in $1 \mathrm{~mL}$ of growth medium and incubated at $37{ }^{\circ} \mathrm{C}$ for $24 \mathrm{~h}$. Then, the cells were treated with PTX injection, ALA-PTX solution or ALA-PTX NPs (PTX $10 \mu \mathrm{M}$ ) for 2,4 or $6 \mathrm{~h}$ at $37^{\circ} \mathrm{C}$. After incubation, the cells were washed three times with cold PBS $(\mathrm{pH}$ 7.4) and lysed with $0.1 \% \operatorname{SDS}(0.1 \mathrm{~mL})$. A volume of $5 \mu \mathrm{L}$ of the lysate was assessed to determine the protein concentration using a Pierce ${ }^{\mathrm{TM}}$ BCA protein assay kit (Thermo Scientific, Waltham, USA). Then, the lysate was extracted with $0.3 \mathrm{~mL}$ acetonitrile and centrifuged at 
$10,000 \mathrm{rpm}$ for $10 \mathrm{~min}$. Then, the resulting supernatant $(20 \mu \mathrm{L})$ was used to measure the content of PTX or ALA-PTX through HPLC analysis. The cellular uptake of PTX injection, ALA-PTX solution and ALA-PTX NPs was calculated using the following formula:

Cellular uptake

$=\frac{\text { The concentration of PTX or ALAPTX in the cells of each well }}{\text { The concentration of total protein in the cells of each well }}$

\section{The Cellular Uptake Pathway of the} ALA-PTX NPs

MCF-7, MCF-7/ADR or HepG2 cells were plated in 12well plates at $2 \times 10^{5}$ cells per well for $24 \mathrm{~h}$ of proliferation. Then, the cell culture medium was replaced with serumfree medium containing inhibitors (listed in Table S2) and ALA-PTX NPs $(10 \mu \mathrm{M})$ and incubated at $37{ }^{\circ} \mathrm{C}$ for $4 \mathrm{~h}$. The other steps were consistent with the experimental steps used for in vitro cellular uptake.

\section{The Intracellular Colocalization}

Dil-loaded ALA-PTX NPs (ALA-PTX $20 \mu \mathrm{M}$ ) were exposed to MCF-7/ADR cells and incubated for $4 \mathrm{~h}$ at $37{ }^{\circ} \mathrm{C}$. Then, the cells were fixed with $4 \%$ paraformaldehyde for $15 \mathrm{~min}$, permeabilized with $0.1 \%$ Triton X-100 for 5 min and blocked with BSA solution.

\section{Clathrin}

The cells were then incubated with the anti-clathrin heavy chain primary antibody overnight at $4{ }^{\circ} \mathrm{C}$. Then, Alexa Fluor 647-labeled secondary antibodies were used to detect the primary antibodies. Five minutes before the end of the incubation, the nuclear dye Hoechst 33342 (20 $\mu \mathrm{g} / \mathrm{mL}$ ) was added to the medium. Finally, cells were observed with CLSM ( $\lambda_{\text {ex }}=549 \mathrm{~nm}$ and $\lambda_{\text {em }}=565 \mathrm{~nm}$ for DiI; $\lambda_{\mathrm{ex}}=350 \mathrm{~nm}$ and $\lambda_{\mathrm{em}}=460 \mathrm{~nm}$ for Hoechst 33342 ; $\lambda \mathrm{ex}=652 \mathrm{~nm}$ and $\lambda \mathrm{em}=668 \mathrm{~nm}$ for Alexa Fluor 647). The cells were then incubated with the anti-clathrin heavy chain antibody overnight at $+4^{\circ} \mathrm{C}$. Finally, Hoechst 33342 and Alexa Fluor 647-labeled secondary antibodies were used to detect the primary antibodies.

\section{Caveolin-I}

The cells were incubated with the anti-caveolin -1-N-terminal (Alexa Fluor 488) antibody (Abcam, Cambridge, MA, USA) overnight at $4{ }^{\circ} \mathrm{C}$. Hoechst 33342 (Beyotime Biotechnology Co., Ltd., Shanghai, China) was used to stain the cell nuclei at a concentration of $20 \mu \mathrm{g} / \mathrm{mL}$ for $10 \mathrm{~min}$ at room temperature. Finally, the cells were observed with CLSM $\left(\lambda_{\mathrm{ex}}=\right.$ $549 \mathrm{~nm}$ and $\lambda_{\mathrm{em}}=565 \mathrm{~nm}$ for DiI; $\lambda_{\mathrm{ex}}=350 \mathrm{~nm}$ and $\lambda_{\mathrm{em}}=$ $460 \mathrm{~nm}$ for Hoechst 33342; $\lambda \mathrm{ex}=495 \mathrm{~nm}$ and $\lambda \mathrm{em}=519$ $\mathrm{nm}$ for Alexa Fluor 488).

\section{Exocytosis Vesicles}

The cells were incubated with primary antibodies (antiRab3A or anti-Rab8A). Finally, Alexa Fluor 647-labeled secondary antibodies were used to detect the primary antibodies. Five minutes before the end of the incubation, the nuclear dye Hoechst 33342 was added to the medium. Finally, cells were observed with CLSM $\left(\lambda_{\mathrm{ex}}=549 \mathrm{~nm}\right.$ and $\lambda_{\mathrm{em}}=565 \mathrm{~nm}$ for DiI; $\lambda_{\mathrm{ex}}=350 \mathrm{~nm}$ and $\lambda_{\mathrm{em}}=460 \mathrm{~nm}$ for Hoechst 33342; $\lambda \mathrm{ex}=652 \mathrm{~nm}$ and $\lambda \mathrm{em}=668 \mathrm{~nm}$ for Alexa Fluor 647).

\section{Lysosomes}

The lysosome tracker in the near infrared light area (was added to the medium $30 \mathrm{~min}$ before the end of the incubation period. Finally, cells were observed with CLSM ( $\lambda$ ex $=636 \mathrm{~nm}$ and $\lambda \mathrm{em}=650 \mathrm{~nm}$ for LysoBrite).

\section{Intracellular Dissolution of ALA-PTX NPs}

MCF-7/ADR cells were seeded at a density of $15 \times 10^{6}$ cells/well in confocal dishes (cover-glass-bottom dish) and incubated at $37{ }^{\circ} \mathrm{C}$ for $24 \mathrm{~h}$. Then, cells were treated with $20 \mu \mathrm{M}$ DiO\&DiI-ALA-PTX NPs in serum-free medium dilutions and incubated at $37^{\circ} \mathrm{C}$ and $5 \% \mathrm{CO}_{2}$ for 2 hours, 6 hours, 12 hours, and 24 hours. Use the FRET SE mode of the laser scanning confocal microscope to detect, delineate the target region (Region of interest, ROI) in the FRET sample image $(\mathrm{n}=15)$, and calculate the FRET efficiency of the ROI. (DiO: $488 \mathrm{~nm}$ excitation, 500-550 nm emission; DiI: $561 \mathrm{~nm}$ excitation, $580-650 \mathrm{~nm}$ emission; FRET: $488 \mathrm{~nm}$ excitation, $580-650 \mathrm{~nm}$ emission) The FRET efficiency was calculated according to the following formula:

$$
\text { FRET ratio }=\frac{\mathrm{I}_{\mathrm{R}}}{\mathrm{I}_{\mathrm{R}+\mathrm{I}_{\mathrm{G}}}}
$$

$I_{R}$ is the fluorescence intensity of DiI at $565 \mathrm{~nm}$, and $I_{G}$ is the fluorescence intensity of $\mathrm{DiO}$ at $501 \mathrm{~nm}$.

\section{In vitro Antitumor Activity}

The in vitro cytotoxicity of ALA-PTX NPs was evaluated in MCF-7/ADR using the sulfonyl rhodamine B (SRB) assay (Sigma-Aldrich, St. Louis, MO, USA). ${ }^{15,16,27}$ MCF-7/ADR cells $\left(6 \times 10^{3}\right.$ cells/well $)$ were seeded in $96-$ 
well plates, incubated for $24 \mathrm{~h}$, and then treated with ALAPTX NPs at $37^{\circ} \mathrm{C}$ for $48 \mathrm{~h}$. The cell viability was determined using SRB, which allowed quantification of the living cells by measuring absorbance at $540 \mathrm{~nm}$ by a $96-$ well plate reader (model 680; Bio-Rad Laboratories Inc., Hercules, CA, USA). The half-maximal inhibitory concentration $\left(\mathrm{IC}_{50}\right)$ was calculated according to the dose-effect curves using GraphPad Prism 8 software.

\section{In vivo Antitumor Activity}

HepG2 and MCF-7/ADR cell lines were used to construct two xenograft tumor models. HepG2 cells or MCF-7/ADR cells $\left(5 \times 10^{6}\right.$ cells in $100 \mu \mathrm{L}$ PBS $)$ were subcutaneously injected into the right armpit of the forelimb of 6-week-old female $\mathrm{BALB} / \mathrm{c}$ nude mice. When the tumors reached $100-300 \mathrm{~mm}^{3}$, the mice were randomly assigned to three groups $(\mathrm{n}=5)$ and treated with $5 \%$ glucose injection, PTX injection $(10 \mathrm{mg} / \mathrm{kg}$ of PTX, iv, q3d $\times 4)$ or ALA-PTX NPs $(40 \mathrm{mg} / \mathrm{kg}$ of ALA-PTX, iv, q3d $\times 4)$ through tail intravenous injection. Tumor size was measured every two days by a caliper, and the tumor volume was calculated by $\mathrm{V}=0.5 \times(\text { width })^{2} \times($ length $)$.

\section{Statistical Analysis}

All experimental data are expressed as the mean $\pm \mathrm{SD}$. The significance among groups was determined using one-way analysis of variance (ANOVA) with Bonferroni post hoc correction for comparisons between individual groups. Statistical significance was shown at $p<0.01$.

\section{Results}

\section{Synthesis and Characterization of ALA-PTX}

ALA-PTX was synthesized by ALA and PTX in a onestep method, coupling ALA and PTX at the 2'-hydroxyl position (Figure 1A). As shown in Figure 1B and C, the characteristic peaks of ALA and PTX could all be found in ALA-PTX. As shown in Figure 1D, the anion mass spectra of ALA-PTX exhibited a single and sharp peak at $\mathrm{m} / \mathrm{z}$ 1112.55, which corresponds to the $[\mathrm{M}-\mathrm{H}]^{-}$. Therefore, ALA-PTX was successfully synthesized, and the yield of ALA-PTX was $68.9 \%$. As shown in Figure 1E, the HPLC chromatogram showed that the peak time of ALA-PTX was $21.4 \mathrm{~min}$. In addition, there were no significant impurity peaks on the chromatogram, and the main peak area accounted for $98.4 \%$ of all peak areas, so the purity of ALA-PTX was about $98.4 \%$.

\section{Preparation and Characterization of ALA-PTX NPs}

ALA-PTX NPs was prepared by using a simple precipitation method (Figure 2A). The drug loading was approximately $90 \%(\mathrm{w} / \mathrm{w})$ with CrEL-free and organic solventfree characteristics. The size of ALA-PTX NPs was approximately $110.7 \pm 1.7 \mathrm{~nm}$. The polydispersity was 0.173 . The zeta potential of the ALA-PTX NPs was $-20.6 \pm 1.5 \mathrm{mV}$. The typical particle size and zeta potential of ALA-PTX NPs were shown in Figure 2B and C. ALAPTX NPs exhibited a homogeneous distribution and good dispersibility in aqueous solution, confirmed by TEM images (Figure 2D). FTIR was used to characterize the groups on the surface of ALA-PTX NPs. As shown in Figure 2E, both ALA and PTX feature groups existed on the surface of ALA-PTX NPs.

\section{In vitro Cellular Uptake of the ALA-PTX NPs}

The in vitro cellular uptake of ALA-PTX NPs was investigated with MCF-7, MCF-7/ADR and HepG2 cell lines. As shown in Figure 3A, compared to PTX injection, ALA-PTX in solution exhibited enhanced uptake by all three cell lines after incubation for 2, 4, or $6 \mathrm{~h}(p<0.05$ or $p<0.01)$, indicating that the functional modification of PTX with ALA increased its cellular uptake. The reason might be that tumor cells are highly dependent on the uptake of exogenous ALA. ${ }^{21}$ In addition, the uptake of ALA-PTX NPs was significantly higher than that of PTX injection and ALA-PTX in solution by all three cell lines $(p<0.05$ or $p<0.01)$, demonstrating that ALA-PTX NPs were taken up by tumor cells at higher levels.

\section{Endocytosis Pathway of the ALA-PTX NPs}

Identifying the NP cellular uptake pathway is important because the cellular uptake pathway can affect the function, intracellular fate and biological response of NPs. ${ }^{28}$ Commonly, cellular uptake pathways include clathrinmediated endocytosis, caveolae-mediated endocytosis, clathrin- and caveolin-independent endocytosis, phagocytosis and macropinocytosis. We used pathway inhibitors to investigate the cellular uptake pathway of ALA-PTX NPs in MCF-7, MCF-7/ADR and HepG2 cells. The results showed that ALA-PTX NPs were taken up by these three 
A

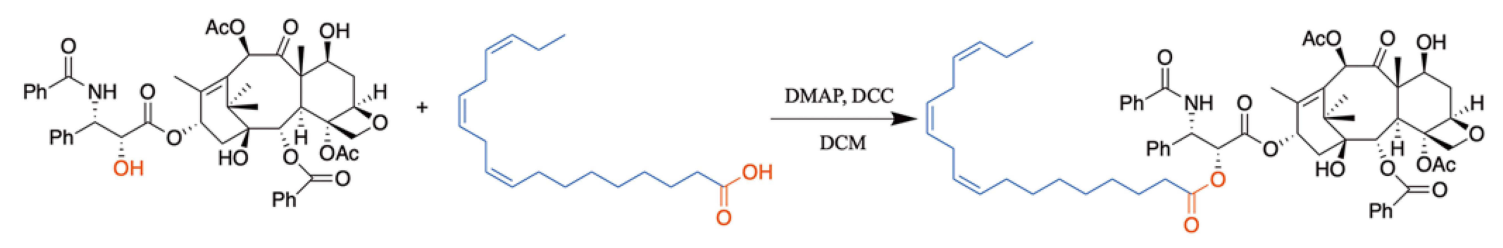

PTX

ALA

ALA-PTX
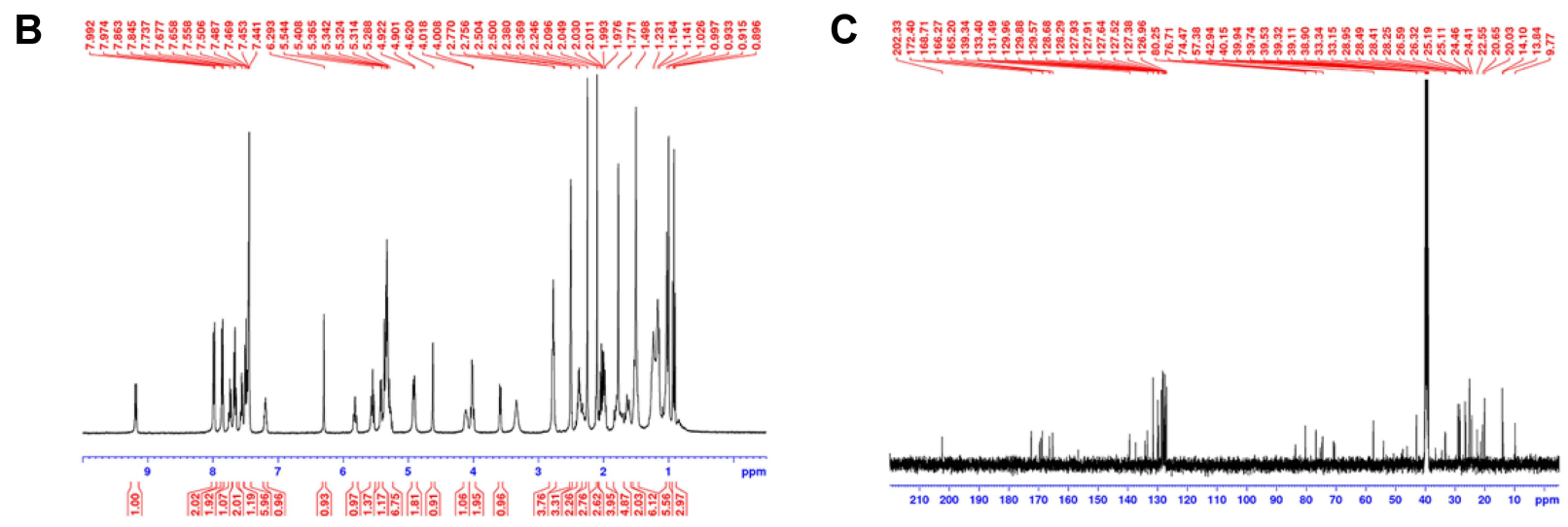

D
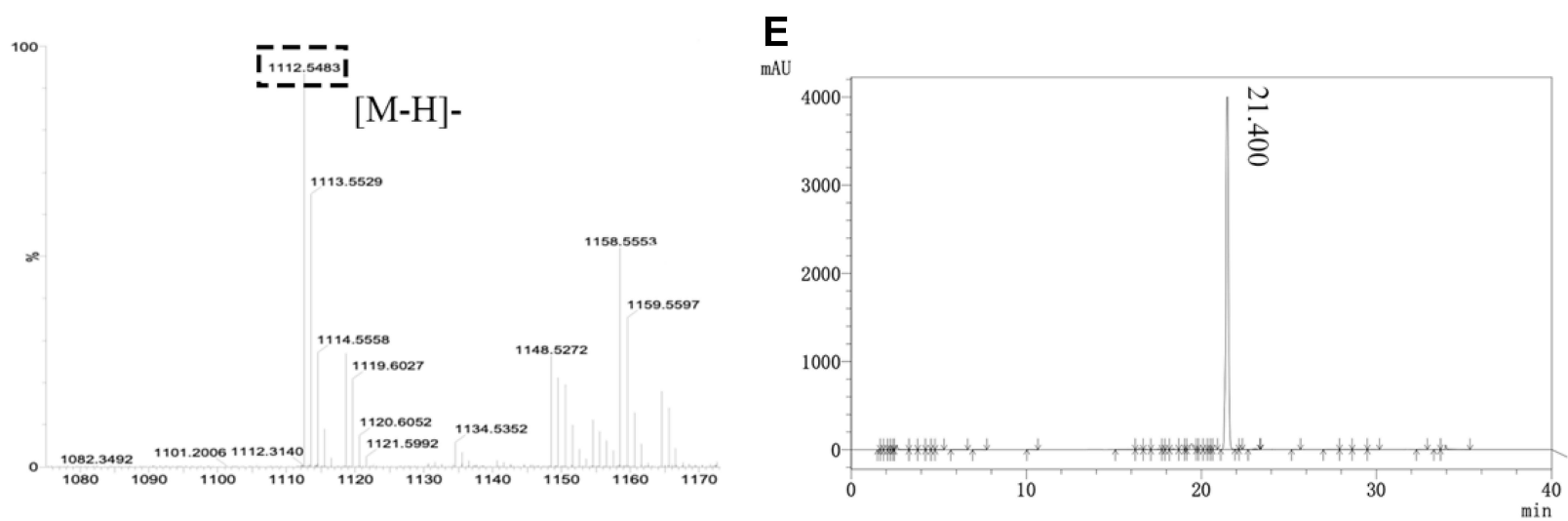

Figure I Synthesis and characterization of ALA-PTX conjugate. (A) Synthetic route of ALA-PTX. (B) 'H NMR spectra of ALA-PTX. (C) ${ }^{13}$ C NMR spectra of ALA-PTX. (D) Anion mass spectra of ALA-PTX. (E) HPLC chromatogram of ALA-PTX.

cell lines through clathrin-mediated endocytosis, as shown in Figure 3B.

\section{Intracellular Behaviors of ALA-PTX NPs}

The co-localization of ALA-PTX NPs with clathrin was investigated, as shown in Figure 4A. The results of immunofluorescence labeling experiments showed that the co-localization of ALA-PTX NPs with clathrin was observed, indicating that ALA-PTX NPs were internalized through the clathrin-mediated endocytosis pathway. Caveolin-1 labeling immunofluorescence results indicated that colocalization of ALA-PTX NPs with caveolin-1 was not observed (Figure 4B). These results indicated that the cellular uptake pathway of ALA-
PTX NPs was independent of caveolin-mediated endocytosis.

Reportedly, NPs that enter cells through clathrinmediated pathways eventually fuse with lysosomes. ${ }^{28}$ To investigate the intracellular transport behavior of ALAPTX NPs, lysosomes were labeled with LysoTracker red, and the colocalization of ALA-PTX NPs with lysosomes was investigated. As shown in Figure 4C, ALA-PTX NPs extensively colocalized with lysosomes, indicating that the ALA-PTX NPs taken up by cells will be delivered into lysosomes.

In addition, the efflux of ALA-PTX NPs was also investigated. Our results indicated that classic secretory vesicles (marked by Rab3) and GLUT4 translocation 
A
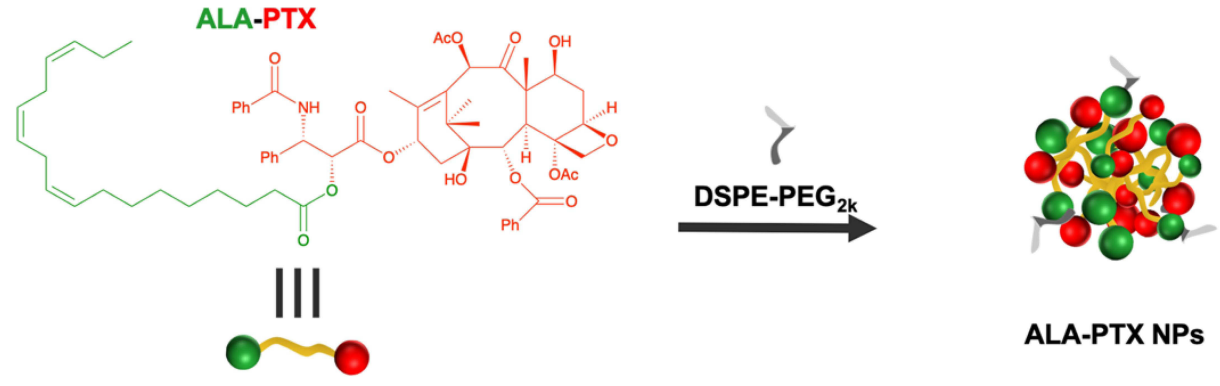

ALA-PTX NPs

B

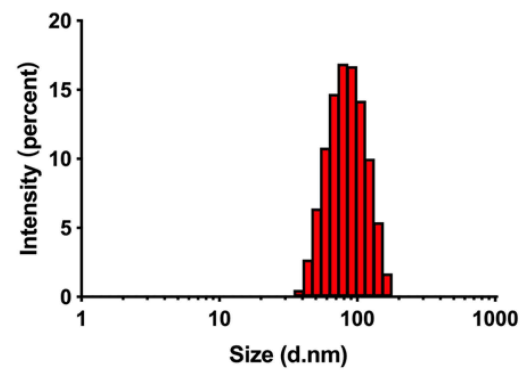

D
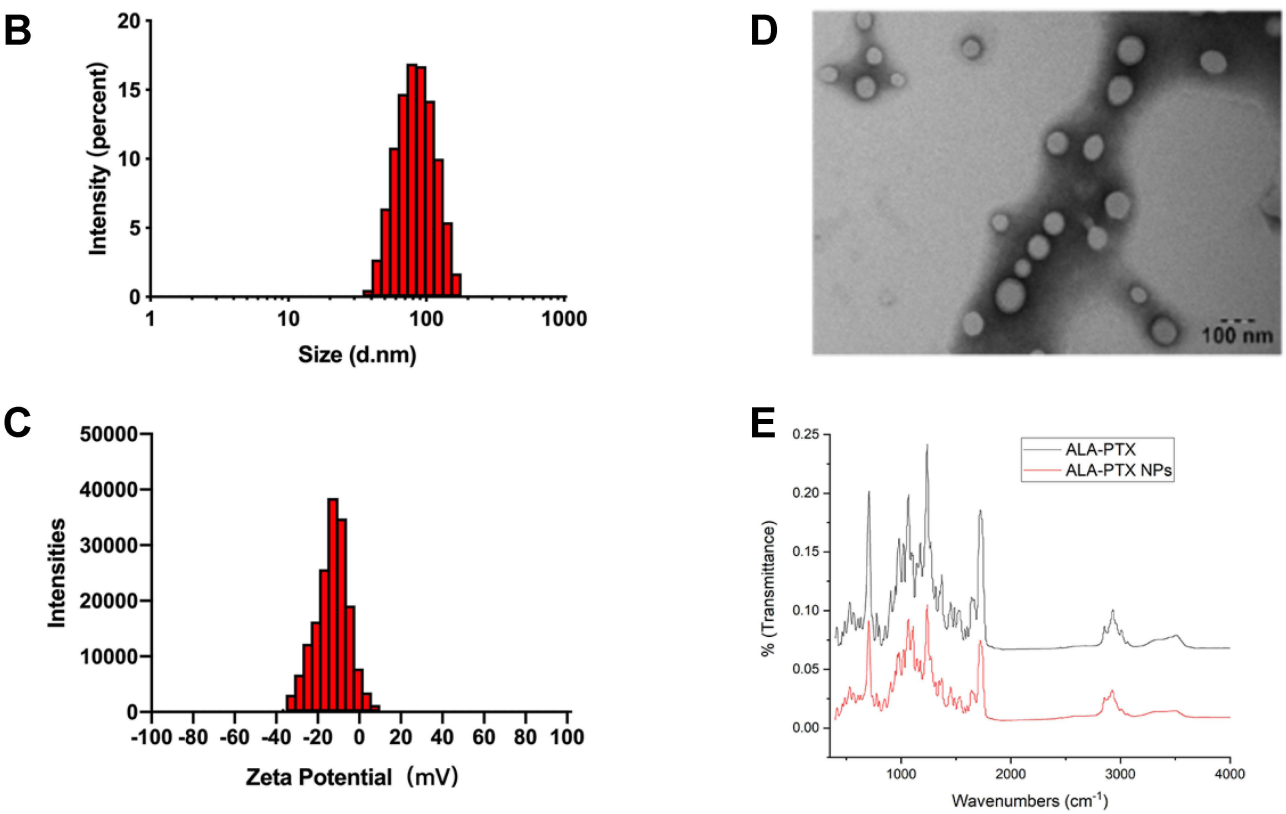

Figure 2 Preparation and characterization of ALA-PTX NPs. (A) Preparation of ALA-PTX NPs. (B) Particle size of ALA-PTX NPs. (C) Zeta potential of ALA-PTX NPs. (D) TEM images of ALA-PTX NPs. (E) FTIR spectra of ALA-PTX and ALA-PTX NPs.

vesicles (marked by Rab8) ${ }^{29}$ had almost no effect on ALA-PTX NP exocytosis, as shown in Figure 4D-E.

\section{Intracellular Dissolution of ALA-PTX NPs}

NPs need to dissolve within the cell to release free drugs to be effective. Therefore, the dissolution of NPs is very important for antitumor efficacy. The intracellular dissolution of ALA-PTX NPs was investigated by Förster resonance energy transfer (FRET) effect. The dyes DiI and $\mathrm{DiO}$ with FRET effect were wrapped in ALA-PTX NPs to prepare DiO\&DiI-ALA-PTX NPs (Figure S1). When DiO\&DiI-ALA-PTX NPs remained intact without dissolution, NPs were excited at $488 \mathrm{~nm}$ (excitation wavelength of $\mathrm{DiO}$ ), and the maximum emission wavelength appeared at $565 \mathrm{~nm}$. If NPs dissolved, when excited with $488 \mathrm{~nm}$, the FRET effect was weakened, the emission light at 501 $\mathrm{nm}$ was enhanced, and the emission light at $565 \mathrm{~nm}$ was weakened.
As shown in Figure 5A, the water dilution of DiO\&DiI-ALA-PTX NPs was excited at $488 \mathrm{~nm}$, the maximum emission wavelength was at $570 \mathrm{~nm}$, and there was a strong FRET effect. The FRET ratio of 0,12 , and 24 $\mathrm{h}$ at room temperature was 0.91 . Therefore, $\mathrm{DiO}$ and $\mathrm{DiI}$ in DiO\&DiI-ALA-PTX NPs would not dissolve in advance within $24 \mathrm{~h}$. DiO\&DiI-ALA-PTX NPs completely dissolved after being diluted with ethanol, and the FRET effect disappeared. Under the excitation of $488 \mathrm{~nm}$, the maximum emission wavelength appeared at $502 \mathrm{~nm}$, and the FRET ratio was 0.11 . This showed that the FRET ratio of DiO\&DiI-ALA-PTX NPs will decrease after the dissolution of DiO\&DiI-ALA-PTX NPs. The FRET ratio can be used to qualitatively describe the intracellular dissolution of DiO\&DiI-ALA-PTX NPs.

DiO\&DiI-ALA-PTX NPs and cells were incubated for $2,6,12$, and $14 \mathrm{~h}$, and the intracellular dissolution of NPs was observed by a confocal microscope. As shown in 

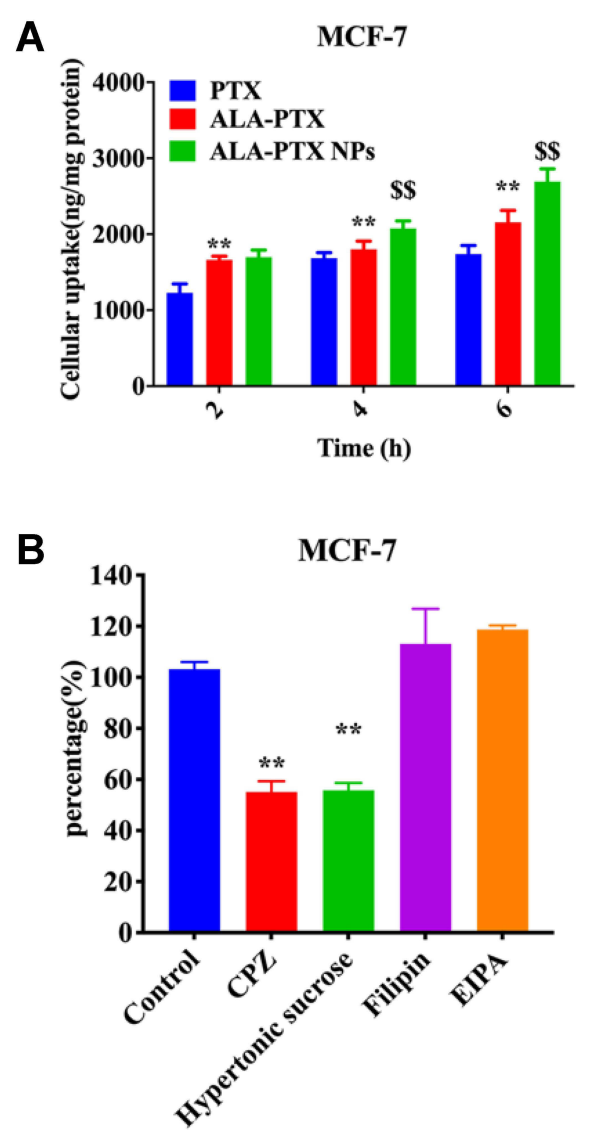
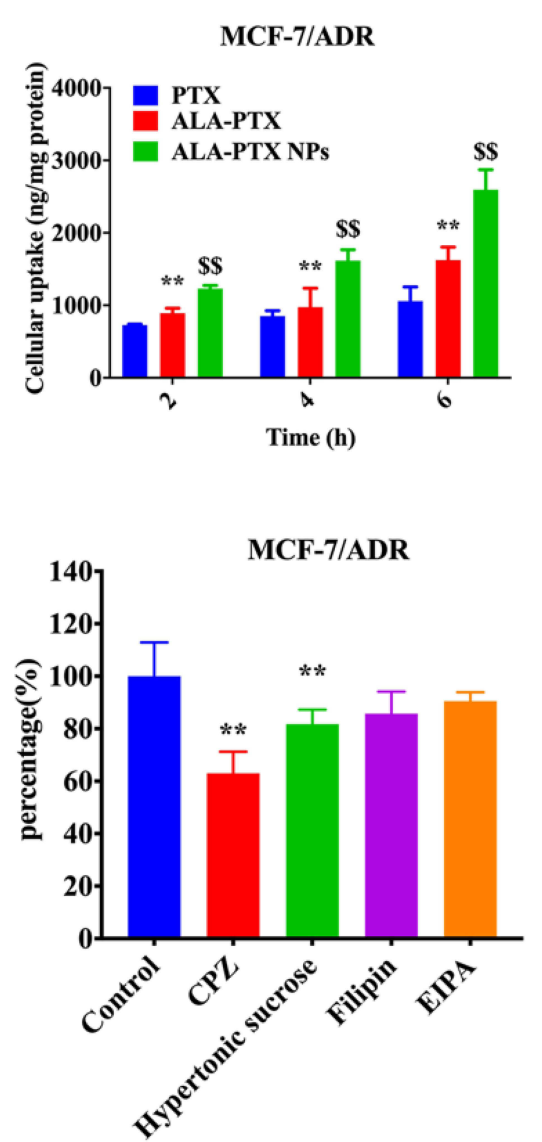
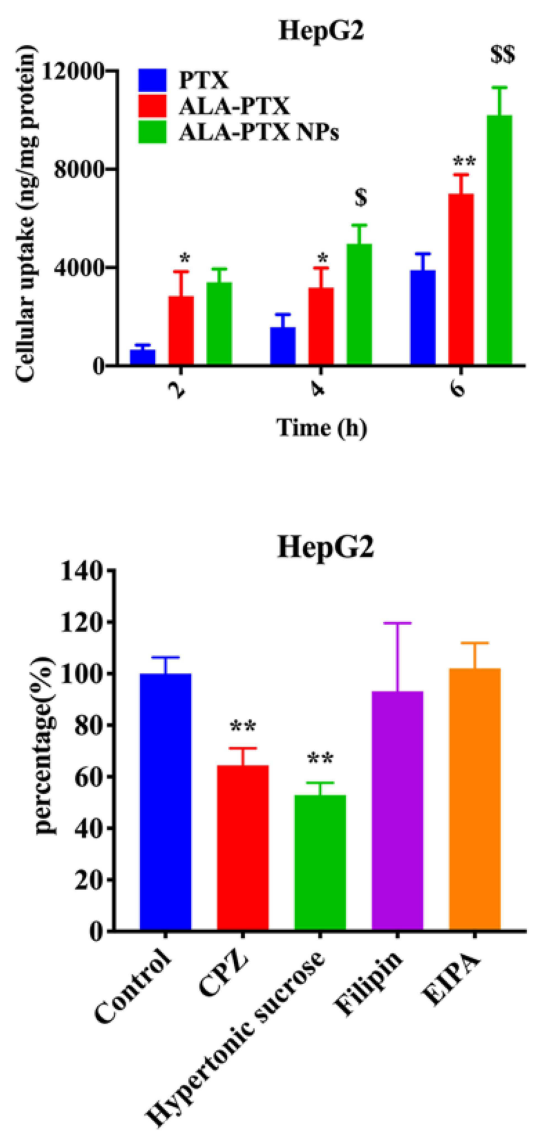

Figure 3 In vitro cellular uptake and antitumor efficacy of ALA-PTX NPs. (A) Uptake of ALA-PTX NPs by MCF-7, MCF-7/ADR and HepG2 cells. *p<0.05, **p<0.0I, compared with PTX injection; ${ }^{\$} p<0.05,{ }^{\$} \$<0.01$, compared with the ALA-PTX solution. (B) Endocytosis inhibitors co-incubated with ALA-PTX NPs in MCF-7, MCF-7/ADR and HepG2 cell to explore the endocytosis pathways. $* * p<0.01$, compared with the cellular uptake of the no inhibitor treatment group (control).

Figure 5B, the green fluorescence was the emission channel of DiO, which represented dissolved NPs, and the red fluorescence was the emission channel of FRET, which represented the completed NPs. At $6 \mathrm{~h}$, the fluorescence intensity of the FRET channel was increased compared to $2 \mathrm{~h}$, and the difference between $12-24 \mathrm{~h}$ and $6 \mathrm{~h}$ was not much. The results showed that the uptake of NPs increased first and then tended to level off. With the extension of the incubation time, the fluorescence intensity of the DiO channel continued to increase, which proved that more and more NPs were dissolved. As shown in Figure 5C, we counted the FRET ratios at different time points, and the results showed that the FRET ratio at 2, 6, 12 and $24 \mathrm{~h}$ was respectively $0.75 \pm 0.10,0.64 \pm 0.03,0.58 \pm 0.08$ and $0.28 \pm 0.05$. With the extension of the incubation time, the FRET ratio decreased significantly $(p<0.01)$, especially at 24 hours. This was because the red fluorescence of the FRET channel remained unchanged and the green fluorescence intensity of DiO increased, so the FRET ratio decreased. Therefore, DiO\&DiI-ALA-PTX
NPs gradually dissolved after cellular uptake, and a large number of disbanded within 24 hours.

\section{In vitro Cytotoxicity}

The in vitro cytotoxicity of ALA-PTX NPs was evaluated in MCF-7/ADR and HepG2 cells, as shown in Table 1. Although the values of $\mathrm{IC}_{50}$ of ALA-PTX NPs were slightly higher than that of PTX injection, the results indicated that there was no significant difference between the $\mathrm{IC}_{50}$ of ALA-PTX NPs and PTX injection in MCF-7/ ADR cells and HepG2 cells $(P>0.05)$.

\section{The in vivo Antitumor Activity of}

\section{ALA-PTX NPs in HepG2 Cell Tumor- and MCF-7/ADR Cell Tumor-Bearing BALB/ \\ c-Nude Mice}

The in vivo antitumor activity of ALA-PTX NPs was investigated in $\mathrm{MCF}-7 / \mathrm{ADR}$ cell tumor-bearing nude mice. As shown in Figure 6A, tumor growth was significantly 


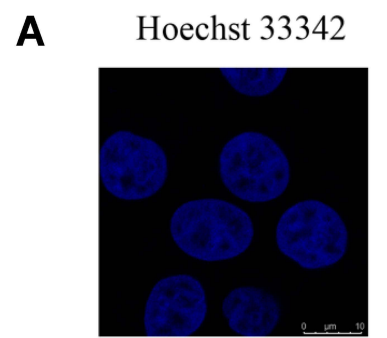

B

Hoechst 33342

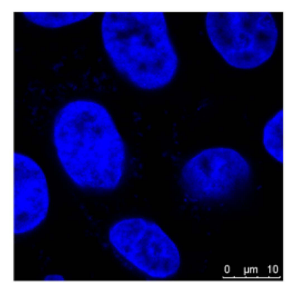

C

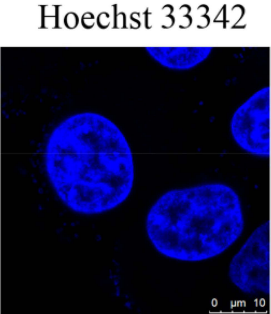

D

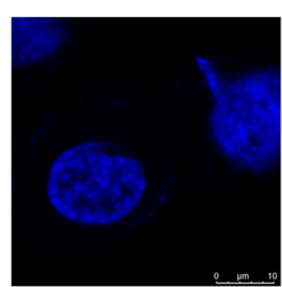

E
Hoechst 33342

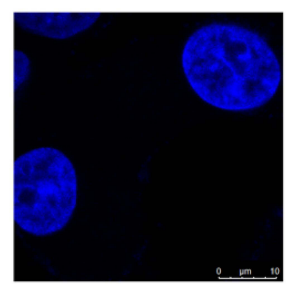

Clathrin

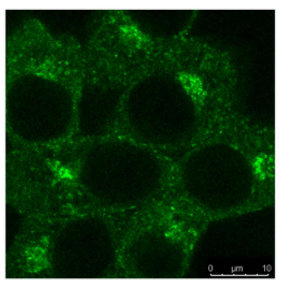

Caveolin-1

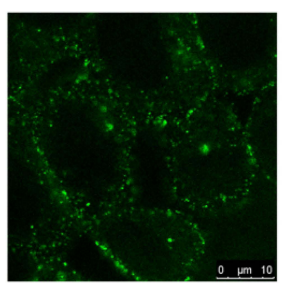

DiI

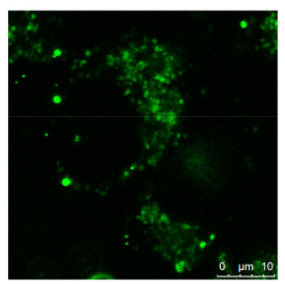

Rab3A

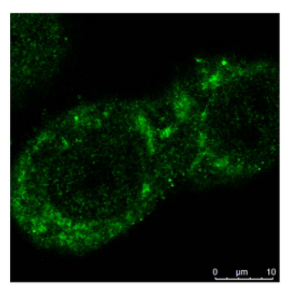

Rab8A

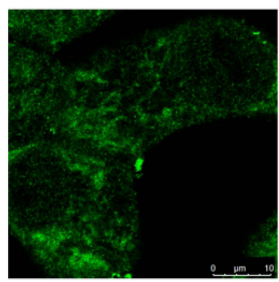

DiI

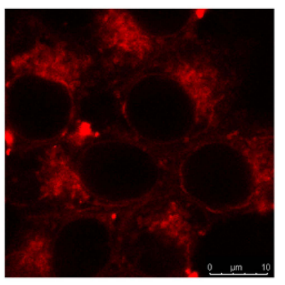

DiI

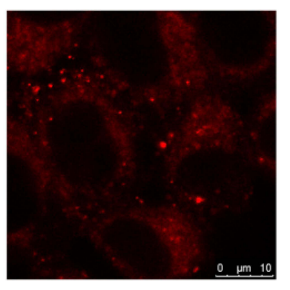

lysosome

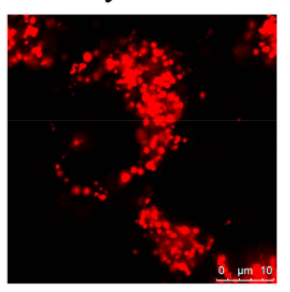

DiI

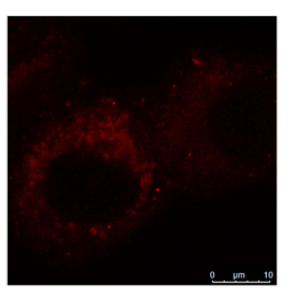

DiI

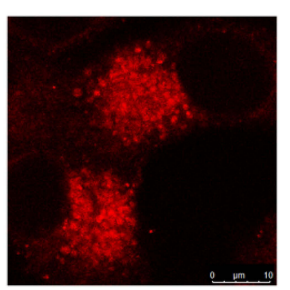

Merge

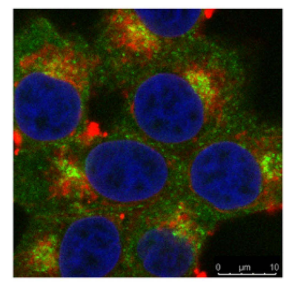

Merge

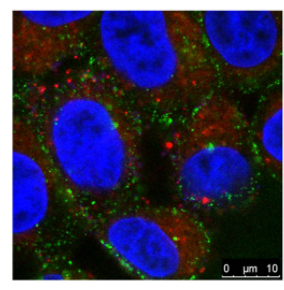

Merge

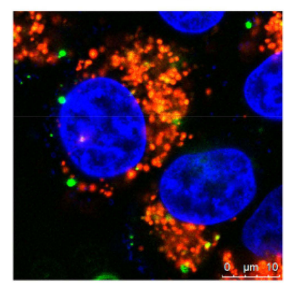

Merge

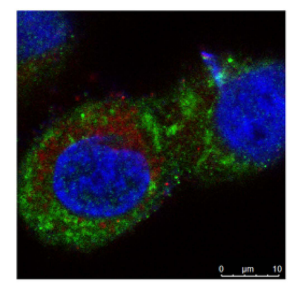

Merge

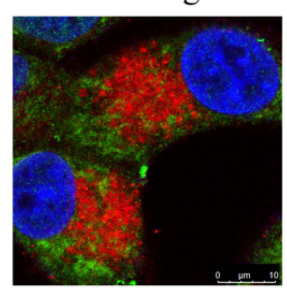

Figure 4 Intracellular behavior of ALA-PTX NPs in HepG2 cells. (A) Co-localization of ALA-PTX NPs and clathrin analyzed by CLSM. (B) Co-localization of ALA-PTX NPs and caveolin-I analyzed by CLSM. (C) Co-localization of ALA-PTX NPs and lysosome analyzed by CLSM. (D) Co-localization of ALA-PTX NPs and Rab3A analyzed by CLSM. (E) Co-localization of ALA-PTX NPs and Rab8A analyzed by CLSM.

inhibited in the PTX injection and ALA-PTX NP groups compared with the control group $(p<0.01)$. The antitumor activity in the ALA-PTX NP group was significantly higher than that in the PTX injection group $(p<0.01)$. The individual tumor growth curve of MCF-7/ADR cell tumor-bearing BALB/c-nude mice was shown in Figure 6D. The mean tumor size 39 days after implantation in the ALA-PTX NP group and PTX injection group was $439 \pm 174$ and $679 \pm$ $63 \mathrm{~mm}^{3}$, respectively, compared with $1266 \pm 278 \mathrm{~mm}^{3}$ in the control group. The corresponding tumor growth inhibition in the ALA-PTX NP group and PTX injection group was $61.3 \%$ and $28.9 \%$ compared with the control group, respectively. Additionally, no obvious weight loss was observed in the mice in the treatment groups and the control group, as shown in Figure 6B. The overall survival of MCF-7/ADR tumor-bearing BALB/c-nude mice in the ALA-PTX NP treatment group was higher than that in PTX injection treatment groups, as shown in Figure 6C. 
A

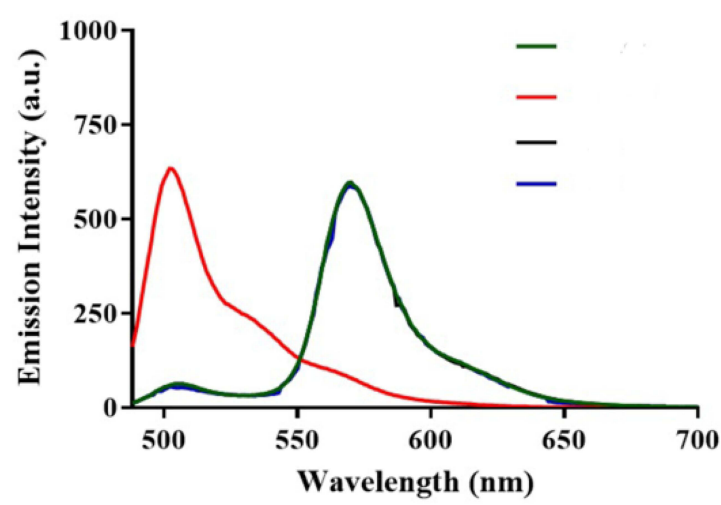

C

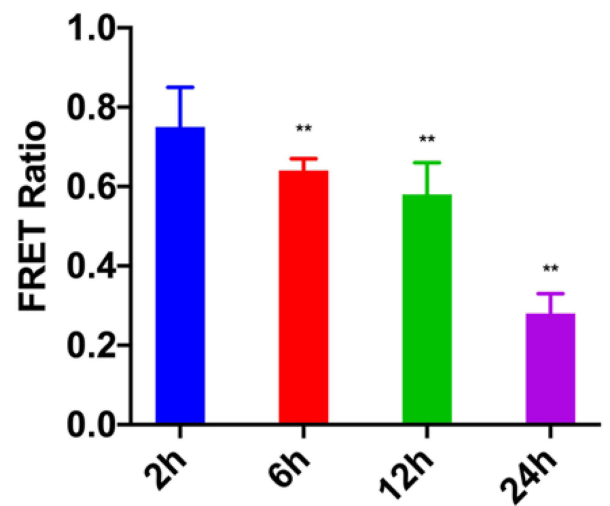

B

DIO

FRET

$2 \mathrm{~h}$

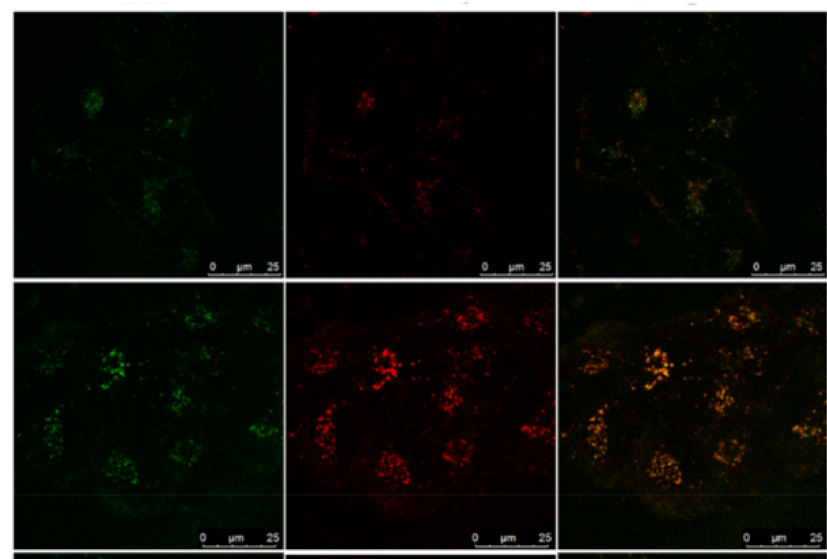

$12 \mathrm{~h}$

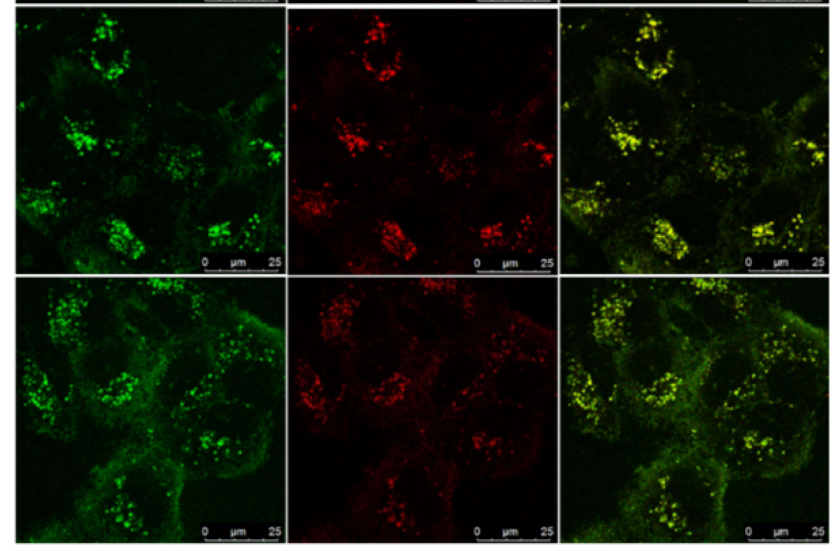

Figure 5 Intracellular dissolution of ALA-PTX NPs. (A) Fluorescence emission spectra of DiO\&Dil-ALA-PTX NPs. Green line: ALA-PTX NPs dissolved in water at Oh; Black line: ALA-PTX NPs dissolved in water at I2h; Blue line: ALA-PTX NPs dissolved in water at 24h; Red line: ALA-PTX NPs dissolved in ethanol at Oh. (B) FRET images of MCF-7/ADR cells incubated with DiO\&Dil-ALA-PTX NPs for 2, 4, 6, 12, 24h. (C) FRET ratio of MCF-7/ADR cells incubated with DiO\&Dil-ALA-PTX NPs for 2, 4, 6, I2, $24 \mathrm{~h}$. ${ }^{*} p<0.0 \mathrm{I}$, compared with the $2 \mathrm{~h}$ group.

Similar results were also observed in the HepG2 cell tumor-bearing nude mouse experiments. As shown in Figure $6 \mathrm{E}$, tumor growth was significantly inhibited in the PTX injection and ALA-PTX NPs treatment groups compared with the control group $(p<0.05$ or $p<0.01)$. The antitumor activity in the ALA-PTX NP treatment group was significantly higher than that in the PTX injection treatment group $(p<0.05)$. The individual tumor growth curve of the HepG2 cell tumor-bearing BALB/c-nude mice was shown in Figure $6 \mathrm{H}$. The mean tumor size at day 35 after implantation in the ALA-PTX NP and

Table I The IC 50 Values $(\mu \mathrm{M})$ of ALA-PTX NPs in MCF-7/ADR and HepG2 Cell Lines

\begin{tabular}{|l|c|c|}
\hline \multirow{2}{*}{ Formulations } & \multicolumn{2}{|c|}{ IC $_{\mathbf{5 0}}(\boldsymbol{\mu M})$} \\
\cline { 2 - 3 } & MCF-7/ADR & HepG2 \\
\hline PTX injection & $1.01 \pm 0.32$ & $0.32 \pm 0.22$ \\
ALA-PTX NPs & $1.30 \pm 0.43$ & $0.60 \pm 0.04$ \\
\hline
\end{tabular}

PTX injection treatment groups was $552 \pm 28$ and $951 \pm$ $217 \mathrm{~mm}^{3}$, respectively, compared with $1688 \pm 437 \mathrm{~mm}^{3}$ in the control group. The corresponding tumor growth inhibition in the ALA-PTX NP and PTX injection treatment groups was $67.3 \%$ and $43.6 \%$ compared with the control group, respectively. Additionally, no obvious weight loss was observed in the mice in the treatment groups and the control group, as shown in Figure 6F. The overall survival of HepG2 tumor-bearing BALB/c-nude mice in the ALA-PTX NP treatment group was higher than that in PTX injection treatment groups, as shown in Figure 6G.

\section{Discussion}

Due to the large demand for exogenous $\alpha$-linolenic acid (ALA) by tumor cells, we used ALA as a small molecule functional material to modify the chemotherapeutic drug PTX and synthesized ALA-PTX. Then we prepared and characterized ALA-PTX NPs. The cellular uptake of 


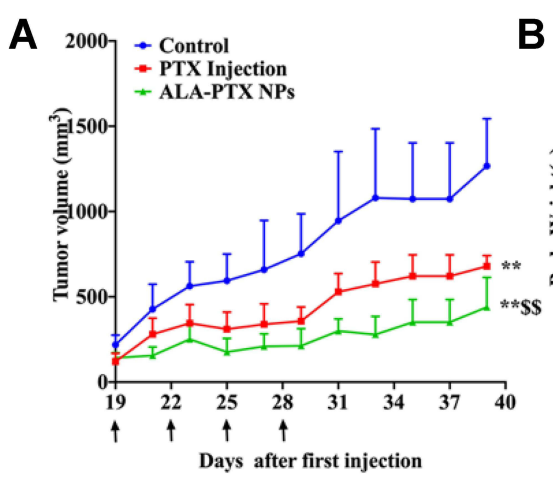

D

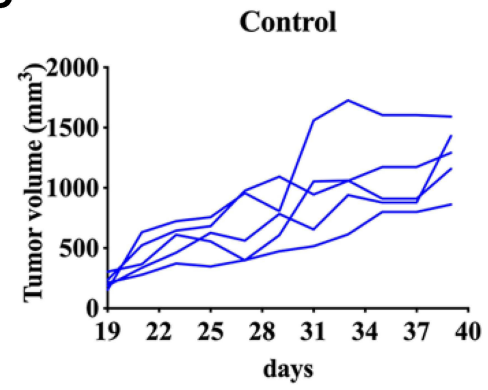

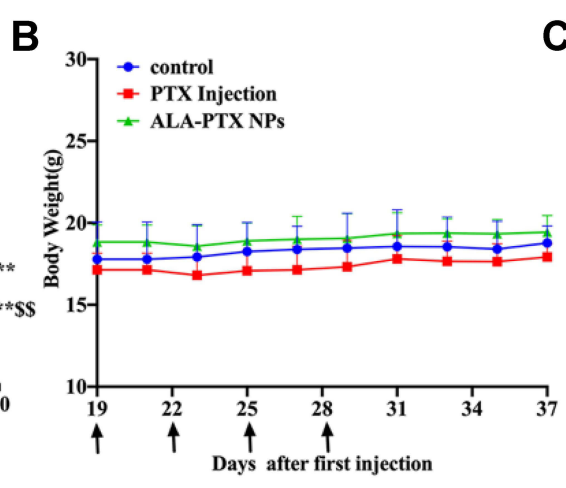

PTX Injection

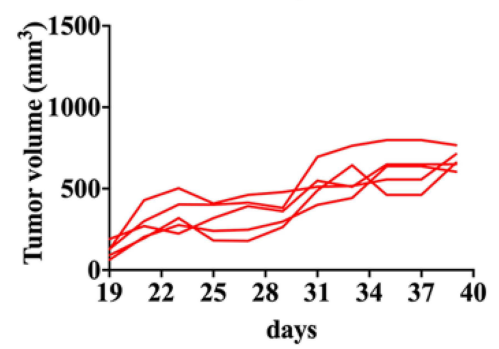

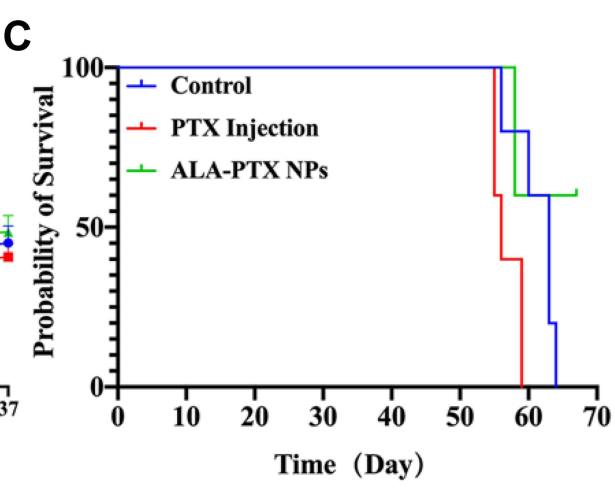

ALA-PTX NPs

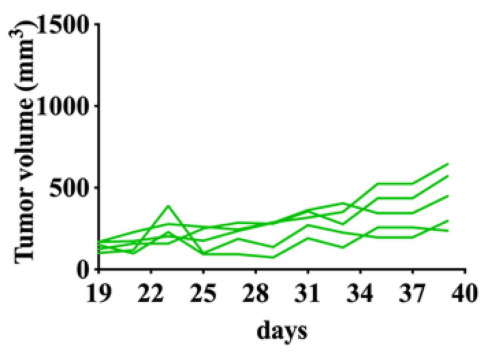

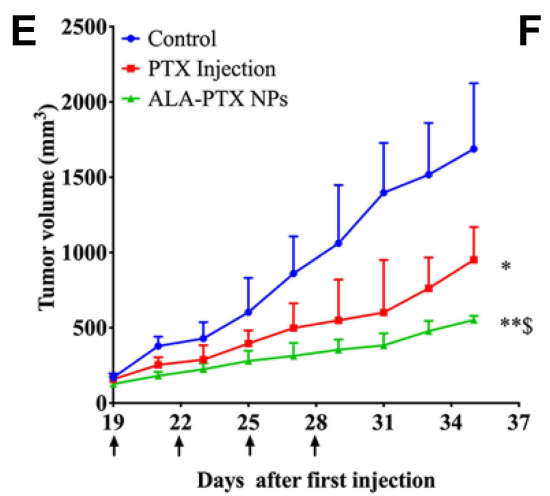

H

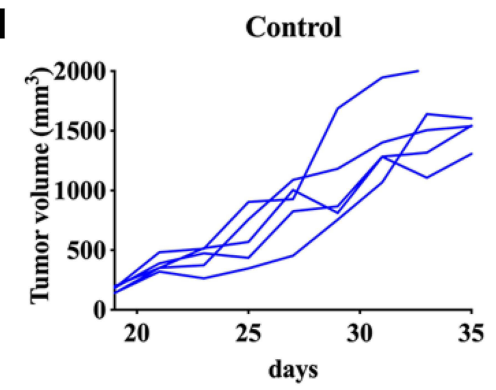

F

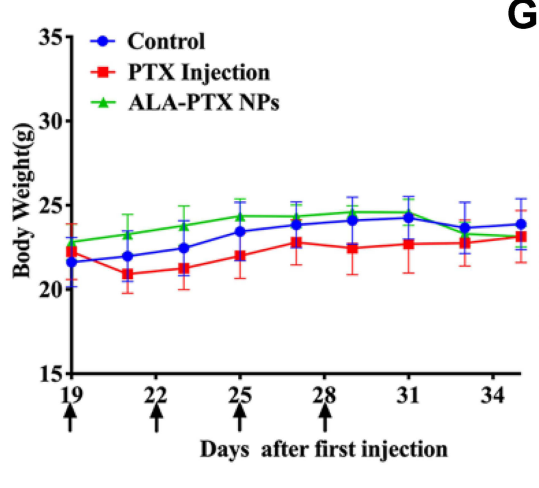

PTX Injection

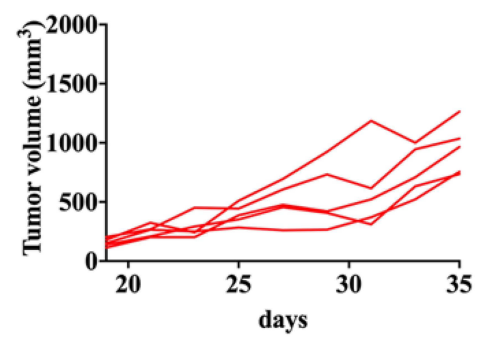

G

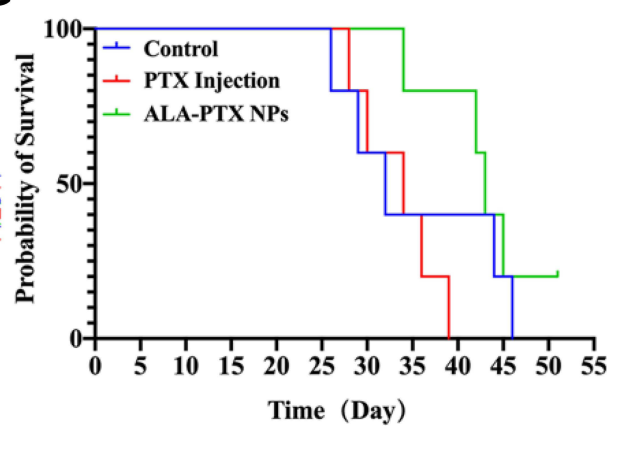

ALA-PTX NPs

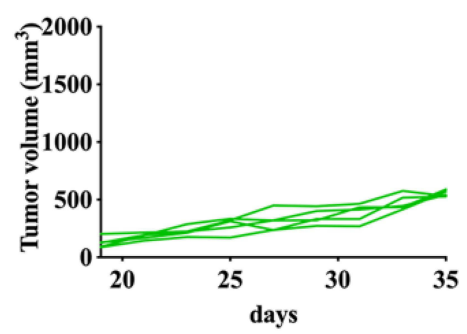

Figure 6 In vivo antitumor activity of ALA-PTX NPs in MCF-7/ADR cell tumor- and HepG2 cell tumor-bearing BALB/c-nude mice treated with 5\% glucose injection, PTX injection ( $10 \mathrm{mg} / \mathrm{kg}$ of PTX, iv, q3d $\times 4$ ) or ALA-PTX NPs $(40 \mathrm{mg} / \mathrm{kg}$ of ALA-PTX, iv, q3d $\times 4)$. (A) Tumor growth curve during treatments of MCF-7/ADR tumor-bearing BALB/c-nude mice. (B) Body weight of MCF-7/ADR tumor-bearing BALB/c-nude mice during treatment. (C) Survival curves of MCF-7/ADR tumor-bearing BALB/c-nude mice. (D) Individual tumor growth curve of MCF-7/ADR tumor-bearing BALB/c-nude mice. (E) Tumor growth curve during the treatments of HepG2 tumor-bearing BALB/ c-nude mice. (F) Body weight of HepG2 tumor-bearing BALB/c-nude mice during treatment. (G) Survival curves of HepG2 tumor-bearing BALB/c-nude mice. (H) Individual tumor growth curve of HepG2 tumor-bearing BALB/c-nude mice. ${ }^{*} p<0.05$, ${ }^{* *} p<0.01$, compared with the control group; ${ }_{p}<0.05$, ${ }^{\$} p<0.01$, compared with the PTX injection group.

ALA-PTX NPs was significantly higher than that of PTX injection by MCF-7, MCF-7/ADR and HepG2 cells. The in vitro and in vivo antitumor activity of
ALA-PTX NPs was confirmed in MCF-7/ADR and HepG2 cell models and tumor-bearing nude mouse models. 
In our previous study, we proposed the prediction criterions which PTX conjugates could form stable NPs in water: when the $\mathrm{X} \log \mathrm{P}$ value of PTX conjugates increased more than 1.0-fold compared to PTX, the conjugate could form stable NPs; otherwise, if its $\delta_{\mathrm{h}}$ and/or $\delta_{\mathrm{p}}$ decreased more than $10 \%$, the conjugate could also form stable NPs. ${ }^{25}$ We calculated the $\triangle \mathrm{X} \log \mathrm{P}$ value of ALAPTX to be 1.82 . The XlogP value of ALA-PTX increased more than 1.0-fold compared to PTX. Therefore, we predicted that ALA-PTX could form stable NPs.

Successfully, we prepared ALA-PTX NPs with high drug loading (approximately 90\%), and without adding a surfactant and organic solvent. Compared with PTX injection, they have higher safety and have bright application prospects. In in vitro anti-tumor efficacy experiments, our results showed that IC50 of ALA-PTX NPs was similar with that of PTX injection. We investigated the maximal tolerable dose (MTD) of ALA-PTX NPs in healthy ICR mice. The results indicated that the MTD of ALAPTX NPs was up to $270 \mathrm{mg} / \mathrm{kg}$ (equimolar to $200 \mathrm{mg} / \mathrm{kg}$ PTX). It was almost eight fold than that of PTX injection $(25 \mathrm{mg} / \mathrm{kg})$, indicating that the safety of ALA-PTX NPs was significantly enhanced compared to PTX injection. Therefore, in in vivo experiments we selected high doses of ALA-PTX NPs (equivalent with $40 \mathrm{mg} / \mathrm{kg}$ of PTX), highlighting that ALA-PTX NPs can be administered at high doses and the advantage of high security. Due to the poor safety, PTX injection could not be administered at a dose of $40 \mathrm{mg} / \mathrm{kg}$. So, we selected the administrated dose of PTX injection was $10 \mathrm{mg} / \mathrm{kg}$ of PTX.

The cellular uptake of ALA-PTX NPs, ALA-PTX solution, and PTX injection was evaluated in MCF-7, MCF-7/ ADR and HepG2 cell lines. The results showed that the uptake of ALA-PTX solution was better than that of PTX injection in these three cell lines, indicating that ALA's chemical modification of PTX could improve cellular uptake. The uptake of ALA-PTX NPs is greater than that of ALA-PTX solution and PTX injection in these three cell lines, which showed that NP dosage forms had advantages in cellular uptake. Recent studies have found that omega-3 PUFAs have the property of crossing the blood-brain barrier through specific receptors. ${ }^{20}$ We speculated that ALA, which is easily transmembrane mediated by receptors, was the reason for the high uptake of ALA-modified chemotherapeutic drugs and their NPs in tumor cells.

After cell internalization, NPs are transported and transported to various intracellular destinations. ${ }^{28}$ If NP cellular uptake occurs via endocytic pathways, NPs are confined within a membrane-lined vesicle, such as an endosome. ${ }^{30}$ These vesicles are transported throughout the cell in complex modes of transport. ${ }^{28}$ Therefore, it is important to understand and explore intracellular behaviors for NPs. We conducted a relatively complete investigation of the intracellular behaviors of ALA-PTX NPs in the aspects of co-localization with endocytic proteins, intracellular transport, and efflux pathways. Our results showed that ALA-PTX NPs were ingested through clathrin-mediated endocytosis, then transferred to lysosomes, and did not excrete cells through classic secretory vesicles and GLUT4 translocation vesicles. ALA-PTX NPs could dissolve in cells to play an antitumor activity.

\section{Conclusion}

In summary, we synthesized and characterized ALA-PTX, and then prepared and characterized ALA-PTX NPs. The cellular uptake of ALA-PTX NPs was significantly higher than that of PTX injection by MCF-7, MCF-7/ADR and HepG2 cells. ALA-PTX NPs were ingested through clathrin-mediated endocytosis, then transferred to lysosomes. ALA-PTX NPs could dissolve in cells to play an antitumor activity. The in vitro and in vivo antitumor activity of ALA-PTX NPs was confirmed in MCF-7/ADR and HepG2 cell models and tumor-bearing nude mouse models. In the future, we will study the in vivo behavior of ALA-PTX NPs, such as experiments on the concentration of nanoparticles in tumor tissue and healthy tissue. ALAPTX NPs developed in this study could provide a new method for the preparation of nano-delivery systems suitable for antitumor therapy that could increase tumor cellular uptake and enhance antitumor activity. The NPs which do not have surfactants and organic solvents, are expected to be clinically converted.

\section{Acknowledgments}

Authors gratefully acknowledge the financial support from the National Key Research and Development Program of China (2017YFA0205600), the National Natural Science Foundation of China (No. 81573360), the Major New Drug Innovation and Development Program of China (2018ZX09721003-004), and the Innovation Team of the Ministry of Education (No. BMU2017TD003).

\section{Disclosure}

The authors report no conflicts of interest in this work. 


\section{References}

1. Shi J, Kantoff PW, Wooster R, et al. Cancer nanomedicine: progress, challenges and opportunities. Nat Rev Cancer. 2017;17:20-37. doi:10.1038/nrc.2016.108

2. Pellico J, Gawne PJ, de Rosales TM. Radiolabelling of nanomaterials for medical imaging and therapy. Chem Soc Rev. 2021;50:3355-3423.

3. Peer D, Karp JM, Hong S, et al. Nanocarriers as an emerging platform for cancer therapy. Nat Nanotechnol. 2007;2:751-760. doi:10.1038/nnano.2007.387

4. Bahrami B, Hojjat-Farsangi M, Mohammadi H, et al. Nanoparticles and targeted drug delivery in cancer therapy. Immunol Lett. 2017;190:64-83.

5. Xin Y, Huang Q, Tang J-Q, et al. Nanoscale drug delivery for targeted chemotherapy. Cancer Lett. 2016;379:24-31. doi:10.1016/j. canlet.2016.05.023

6. Sreekanth V, Bajaj A. Recent advances in engineering of lipid drug conjugates for cancer therapy. ACS Biomater Sci Eng. 2019;5:4148-4166. doi:10.1021/acsbiomaterials.9b00689

7. Karaosmanoglu S, Zhou M, Shi B, et al. Carrier-free nanodrugs for safe and effective cancer treatment. J Control Release. 2021;32 9:805-832. doi:10.1016/j.jconrel.2020.10.014

8. Zheng Y, Ying X, Su Y, et al. Kinetically-stable small-molecule prodrug nanoassemblies for cancer chemotherapy. Int $J$ Pharm 2021;597:120369. doi:10.1016/j.ijpharm.2021.120369

9. Wang $\mathrm{H}$, Xie $\mathrm{H}$, Wang J, et al. Self-assembling prodrugs by precise programming of molecular structures that contribute distinct stability, pharmacokinetics, and antitumor efficacy. Adv Funct Mater. 2015;25:4956-4965. doi:10.1002/adfm.201501953

10. Fang T, Dong Y, Zhang X, et al. Integrating a novel SN38 prodrug into the PEGylated liposomal system as a robust platform for efficient cancer therapy in solid tumors. Int $J$ Pharm. 2016;512:39-48. doi:10.1016/j.ijpharm.2016.08.036

11. Zhang T, Li M, Yang R, et al. Therapeutic efficacy of lipid emulsions of docetaxel-linoleic acid conjugate in breast cancer. Int J Pharm. 2018;546:61-69. doi:10.1016/j.ijpharm.20 18.05.032

12. Zeng J, Li C, Duan X, et al. PEGylation of lipophilic SN38 prodrug with DSPE-mPEG (2000) versus cremophor EL: comparative study for intravenous chemotherapy. Drug Deliv. 2019;26:354-362. doi:10.1080/10717544.2019.1587045

13. Schulze M, Minihane A, Saleh R, et al. Intake and metabolism of omega-3 and omega- 6 polyunsaturated fatty acids: nutritional implications for cardiometabolic diseases. Lancet Diabetes Endocrinol. 2020;8:915-930. doi:10.1016/S2213-8587 (20)30148-0

14. Panagiotopoulos AA, Kalyvianaki K, Castanas E, et al. Eicosanoids in prostate cancer. Cancer Metastasis Rev. 2018;37:237-243. doi:10. 1007/s10555-018-9750-0

15. Ke XY, Zhao BJ, Zhao X, et al. The therapeutic efficacy of conjugated linoleic acid - paclitaxel on glioma in the rat. Biomaterials. 2010;31:5855-5864. doi:10.1016/j.biomaterials.20 10.03.079

International Journal of Nanomedicine

\section{Publish your work in this journal}

The International Journal of Nanomedicine is an international, peerreviewed journal focusing on the application of nanotechnology in diagnostics, therapeutics, and drug delivery systems throughout the biomedical field. This journal is indexed on PubMed Central, MedLine, CAS, SciSearch ${ }^{\mathbb{R}}$, Current Contents ${ }^{\mathbb{R}} /$ Clinical Medicine, $^{2}$
16. Zhong T, Yao X, Zhang S, et al. A self-assembling nanomedicine of conjugated linoleic acid-paclitaxel conjugate (CLA-PTX) with higher drug loading and carrier-free characteristic. Sci Rep. 2016;6:36614. doi:10.1038/srep36614

17. Golanski J, Szymanska P, Rozalski M. Effects of omega-3 polyunsaturated fatty acids and their metabolites on haemostasis-current perspectives in cardiovascular disease. Int J Mol Sci. 2021;22(5):2394. doi:10.3390/ijms22052394

18. Zaloga G. nNarrative review of -3 polyunsaturated fatty acid supplementation upon immune functions, resolution molecules and lipid peroxidation. Nutrients. 2021;13(2):662. doi:10.3390/nu13020662

19. Macaron T, Giudici K, Bowman G, et al. Associations of Omega-3 fatty acids with brain morphology and volume in cognitively healthy older adults: a narrative review. Ageing Res Rev. 2021;67:101300. doi:10.1016/j.arr.2021.101300

20. Cater R, Chua G, Erramilli S, et al. Structural basis of omega-3 fatty acid transport across the blood-brain barrier. Nature. 2021;595:315-319. doi:10.1038/s41586-021-03650-9

21. Gu Z, Suburu J, Chen H, et al. Mechanisms of omega-3 polyunsaturated fatty acids in prostate cancer prevention. Biomed Res Int. 2013;2013:824563. doi:10.1155/2013/824563

22. Truan JS, Chen JM, Thompson LU. Flaxseed oil reduces the growth of human breast tumors (MCF-7) at high levels of circulating estrogen. Mol Nutr Food Res. 2010;54:1414-1421. doi:10.1002/mnfr.200900521

23. Abouelela SH, Prasse KW, Farrell RL, et al. Effects of D, L-2-difluoromethylornithine and indomethacin on mammary tumor promotion in rats fed high n-3 and/or n-6 fat diets. Cancer Res. 1989;49:1434-1440.

24. Brown MD, Hart CA, Gazi E, et al. Promotion of prostatic metastatic migration towards human bone marrow stoma by Omega 6 and its inhibition by Omega 3 PUFAs. $B r J$ Cancer. 2006;94:842-853. doi:10.1038/sj.bjc.6603030

25. Zhong T, Hao YL, Yao X, et al. Effect of XlogP and Hansen solubility parameters on small molecule modified paclitaxel anticancer drug conjugates self-assembled into nanoparticles. Bioconjug Chem. 2018;29:437-444. doi:10.1021/acs.bioconjchem.7b00767

26. Duan XC, Yao X, Zhang S, et al. Antitumor activity of the bioreductive prodrug 3-(2-nitrophenyl) propionic acid-paclitaxel nanoparticles (NPPA-PTX NPs) on MDA-MB-231 cells: in vitro and in vivo. Int J Nanomedicine. 2019;14:195-204. doi:10.2147/IJN.S186556

27. Du R, Zhong T, Zhang WQ, et al. Antitumor effect of iRGD-modified liposomes containing conjugated linoleic acid-paclitaxel (CLA-PTX) on B16-F10 melanoma. Int J Nanomedicine. 2014;9:3091-3105.

28. Donahue ND, Acar H, Wilhelm S. Concepts of nanoparticle cellular uptake, intracellular trafficking, and kinetics in nanomedicine. $A d v$ Drug Deliv Rev. 2019;143:68-96.

29. Ding L, Zhu X, Wang Y, et al. Intracellular fate of nanoparticles with polydopamine surface engineering and a novel strategy for exocytosis-inhibiting, lysosome impairment-based cancer therapy. Nano Lett. 2017;17:6790-6801. doi:10.1021/acs.nanolett.7b03021

30. Kim CS, Li X, Jiang Y, et al. Cellular imaging of endosome entrapped small gold nanoparticles. MethodsX. 2015;2:306-315. doi:10.1016/j.mex.2015.06.001
Journal Citation Reports/Science Edition, EMBase, Scopus and the Elsevier Bibliographic databases. The manuscript management system is completely online and includes a very quick and fair peer-review system, which is all easy to use. Visit http://www.dovepress.com/ testimonials.php to read real quotes from published authors. 